\title{
POLLUTION SURVEILLANCE AND DATA ACQUISITION USING MULTISPECTRAL REMOTE SENSINGl
}

Chester T. Wezernak and Fabian C. Polcyn ${ }^{2}$

\section{ABSTRACT}

Large scale water resource investigations and effective pollution surveillance programs require the development of additional instrumentation and techniques to supplement existing methods of data acquisition. As a result, interest is growing in the concept of remote sensing. Described in this paper is the multispectral sensor concept and its application in water resource studies.

KEY WORDS: remote sensing; water pollution surveillance; industrial wastes; water pollution control; instrumentation

\section{INTRODUCTION}

The current effort to bring all existing pollution under effective control, and to enhance the quality of our water resources, has provided an added incentive for the development of instrumentation and techniques for detecting and identifying sources of water pollution. As a consequence, impressive gains have been made in recent years in the development of analytical techniques and continuous monitoring instrumentation. However, in view of the scope, dynamic nature, and dimension of the pollution control problem, additional techniques are required to detect sources of pollution, identify the pollutant, and to define dispersion patterns. This is particularly true for large water masses, coastal areas, and large river systems. As a consequence, interest is growing in the concept of remote sensing.

The term remote sensing refers to the acquisition of information about objects or phenomena in our environment through the use of sensory devices at positions away from the subject under investigation. In this investigation the term is restricted to the use of an airborne system and measurement of interactions in the ultraviolet, visible, and infrared regions of the electromagnetic spectrum.

The amount of information potentially obtainable from remote measurement of electromagnetic energy is quite large. As Colwe11, et al. (1963) have emphasized, only four interactions are possible when a photon of any specific energy strikes a substance. The energy may be absorbed, emitted, scattered, or reflected. Therefore, in principle, information can be obtained regarding an object provided, of course, that the instrumentation used is capable of defining spectral properties in sufficient detail.

The data acquisition needs in the water pollution control field include information regarding the following:

1 Paper No. 70081 of the Water Resources BulZetin (Journal of the American Water Resources Association). Discussions are open unti1 June 1, 1971.

2Respectively, Assistant Professor, Department of Environmental and Industrial Health, University of Michigan, Ann Arbor, Michigan; and Research Engineer, Infrared and Optics Laboratory, Willow Run Laboratories, University of Michigan, in Arbor, Michigan. 
1. materials on the surface of the water,

2. materials suspended in the water, and

3. materials dissolved in the water.

Some of this information is at times expressed in broad-term parameters such as suspended solids, total carbon, oils, etc.; and also, of course, in very specific chemical terms. Additionally, there is a need for information of a strictly physical nature; such as temperature or data regarding pollution dispersion patterns. The information needs are clearly quite extensive and as a result cannot be fully realized with a single sensor system.

The present report deals with one sensor system and presents selected examples of problems wherein remote sensing can offer a number of unique advantages.

\section{MULTISPECTRAL SENSOR SYSTEM}

The instrumental technique used in this investigation involved the use of the multispectral sensor developed at the University of Michigan (Holter, 1967).

The University of Michigan has pioneered the development of a passive multichannel scanner system which is capable of sensing reflected and emitted energy simultaneously in a number of narrow spectral intervals in the ultraviolet, visible and infrared region of the solar spectrum. The system basically consists of a spectrometer for dispersing the radiation spectrally and filtered detector arrays placed at the focal points of a double ended optical-mechanical scanner. Figure 1 shows schematically the system operation. The scanner uses reflective optics to collect energy over a wide spectral band between ultraviolet and thermal infrared. The entrance slit of the spectrometer is placed at the focal plane of the scanner to insure that space and time simultaneity is achieved for all channels between .4 and $1 \mu$. Filtered detectors for the infrared region and the use of short electronic time delays provide coincident data for the region beyond $1 \mu$. Additional detectors and filters can be provided for the ultraviolet region. Signals from the detectors are stored on magnetic tape for later image reconstruction and data processing. As an integral feature of the system, reference lamps, an input proportional to the sun energy, and adjustable temperature plates are viewed each revolution of the scanner mirror. Thus for the first time (with an optical-mechanical scanner) reproducible data can be obtained which is referenced to known radiation sources and is capable

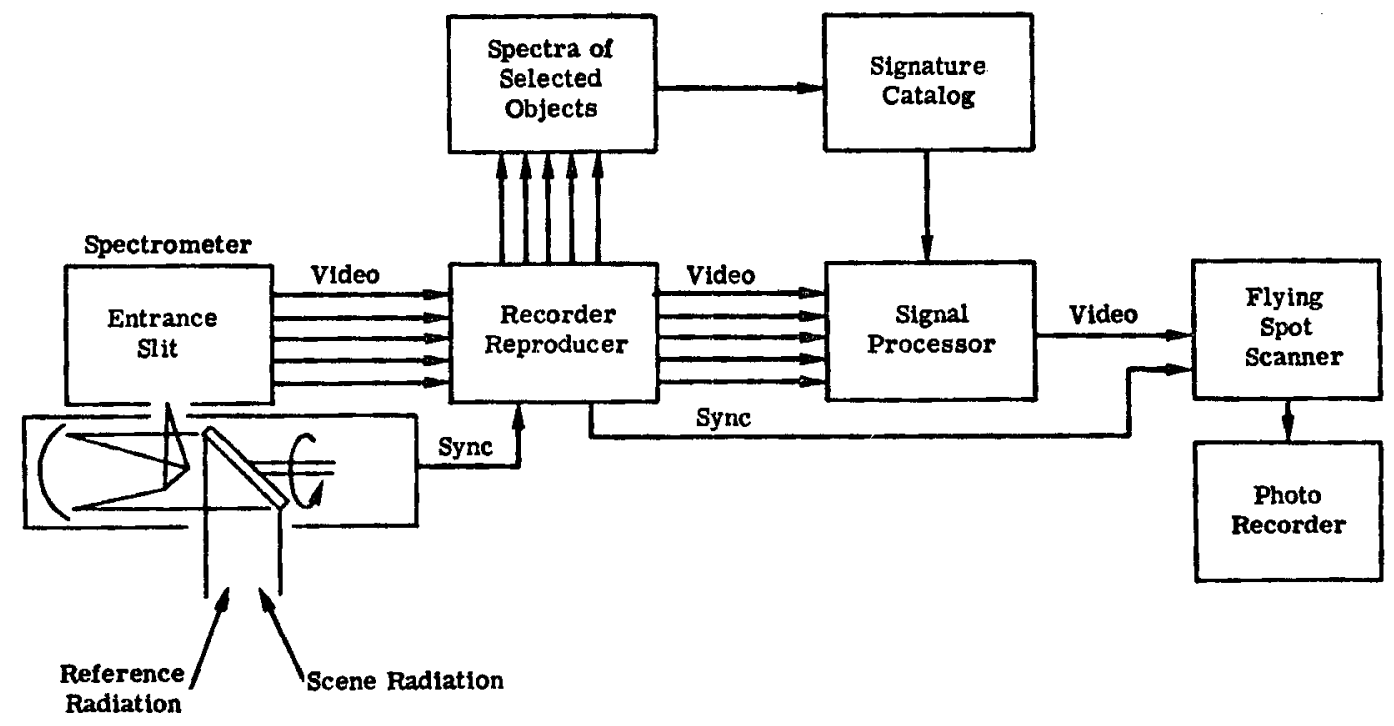

FIGURE 1. Schematic of multispectral scanner and data processor. 
of accounting for changes in solar illumination. These controlled points in each scan line permit the use of sophisticated electronic pattern recognition techniques with both analog and digital computers (Nalepka, 1970).

The generation of a spectrum for each element permits machine recognition of classes of objects whose spectral characteristics are similar within certain electronic decision levels. As part of on-going research, the University of Michigan is engaged in a spectral measurement program to identify the spectral characteristic of objects and determine their mean and variance statistics for a class of similar objects. In the field of water pollution control it is believed that remote spectral measurements can be used to detect and identify effluent discharges.

In order to determine spectral characteristics, the airborne data recorded on magnetic tape is electronically digitized and then stored in a computer. The analog to digital converter consists of a high accuracy (13 bit and sign) successive approximation converter capable of providing samples in 10 microseconds. The data is formated and coded into 48 bit words for a CDC-1604 computer. Special computer programs have been written to edit the digital tapes and format the data for use with FORTRAN programs. Selected portions of the objects mapped are used as training areas for which the spectral characteristics are computed. Once computed, a computer generated spectral curve can be made that is referenced to the incoming solar spectrum or to other objects in the scene. In some cases, ground reflectance standards are used to provide absolute reflectance calibration.

The spectral properties are then available for use with the computer to perform spectral matching operations to enhance the detection of selected objects of similar properties or to map the different concentration of an effluent.

The electronic format of the data also permits a voltage sampling operation (for any selected band) in order to display small density differences. For example, the mixing zones of a thermal discharge into a river or lake can be studied readily by quantizing the data in the 8-13.5 $\mathrm{\mu}$ band into one half degree steps, color coding each step, and combining the set into one color composite display. Each color represents the isotherm for a chosen temperature level. The use of two adjustable reference plates in the scanner system insures optimum recording and accurate reconstruction of the apparent temperature patterns.

Many other computer programs have been devised and are employed in processing the multispectral data for particular applications.

\section{APPLICATIONS}

Detection and identification of any object is possible only if there is a significant contrast in physical or chemical properties between the object being examined and its background. In the case of effluent discharges into the aquatic environment; from power plants, industrial sources, and municipal sources, there is normally some significant difference between the effluent and the receiving body of water which can be detected by the system described above. Additionally, the very large discrimination capability of the sensor system allows for the characterization of the target, at least in terms of broad parameters. Described in the sections which follow are selected examples which serve to define areas of application wherein remote sensing technology offers a number of advantages.

\section{TEMPERATURE ANALYSIS}

Temperature is one parameter which is closely related to water pollution and is of interest in virtually all studies dealing with the aquatic environment. Fortunately, surface determinations can readily be made in the $8-13.5 \mu$ region throughout the day or night to an accuracy of $0.5^{\circ} \mathrm{C}$. This ability has been clearly demonstrated by a number of investigators. However, the important difference between what has been reported in the past and the present investigation, centers around the in- 
creased instrument capability due to the calibration and correlation with other portions of the spectrum.

An added feature of the University of Michigan system is the calibration lamp and controlled thermal sources that are recorded for each scan line. This feature allows for greater sensitivity in temperature determinations and thermal contour development.

Shown in Figure 2 is a portion of a thermal plune discharging from a power plant. The general distribution of the warm water being discharged is clearly visible in the lower panel. Using the computer processing technique referred to earlier, the airborne thermal data was used to develop temperature contours. Shown in the figure are selected temperature levels in the plume area.

In addition to applicability in thermal pollution studies; the speed, mobility, and ability to completely and virtually instantaneously map large water areas can be of great importance in a variety of other water resource investigations. For example, the movement of water masses can be followed and defined. This can be accomp1ished in terms of a number of physical-chemical characteristics. However, if in a given situation a temperature difference exists between the two water bodies, monitoring can easily be done in the 8-13.5 $\mu$. range during hours of daylight or darkness. An example is shown in Figure 3. In this situation the progression of a cold water release from an upstream dam was defined. The dark area represents water approximately $3^{\circ} \mathrm{C}$ colder than the light areas shown.

\section{EFFLUENT OUTFALLS}

The ability to detect small temperature differences can also be used to locate effluent outfalls including submerged outfalls; provided of course, that the volumes discharged are of sufficient magnitude to alter the temperature of the receiving waters. Detection on the basis of other effluent characteristics is also possible. However, in virtually all cases there is a temperature difference between the effluent and the receiving body of water which can serve as a basis for detection. Monitoring in the 8-13.5 $\mu$ region of the electromagnetic spectrum can be done during the day or night and hence provides an expanded capability for pollution surveillance.

Shown in Figure 4 (lower panel) is the plume from a 3 M.G.D. municipal waste treatment plant discharging from a submerged outfall. In this case, the variations in emitted power level are probably due to a change in temperature and emissivity due to a change in water quality.

\section{UNDERWATER FEATURES}

It is generally recognized that in examining any scene, certain components of a target can be best defined by their response within selected narrow spectral bands. This fact is clearly illustrated in Figure 5 which demonstrates the capability of the system in locating and mapping underwater features in clear water. The imagery shown was obtained at the south end of Biscayne Bay in Florida and shows maximum overall light penetration in seawater in the 0.55 to $0.58 \mu$ bandwidth, with Ceasar Creek clearly defined in the 0.62 to $0.68 \mu$ bandwidth (Polcyn and Rollin, 1969).

\section{INDUSTRIAL EFFLUENTS}

As indicated earlier, an important feature of the instrumental system used in this investigation is the ability to examine a scene in 17 bands distributed between 0.3 and $13.5 \mathrm{H}$. This means that in addition to the detection capability referred to above, the system serves as a 17 channel spectrometer with electronic processing features. Shown in Figures 6-9 are a few examples of multispectral imagery of industrial discharges. 

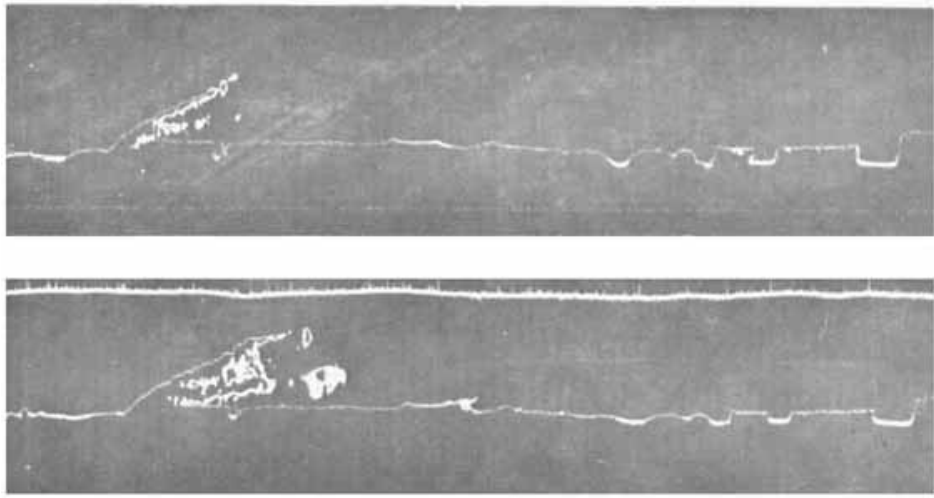

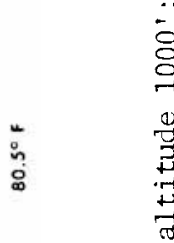
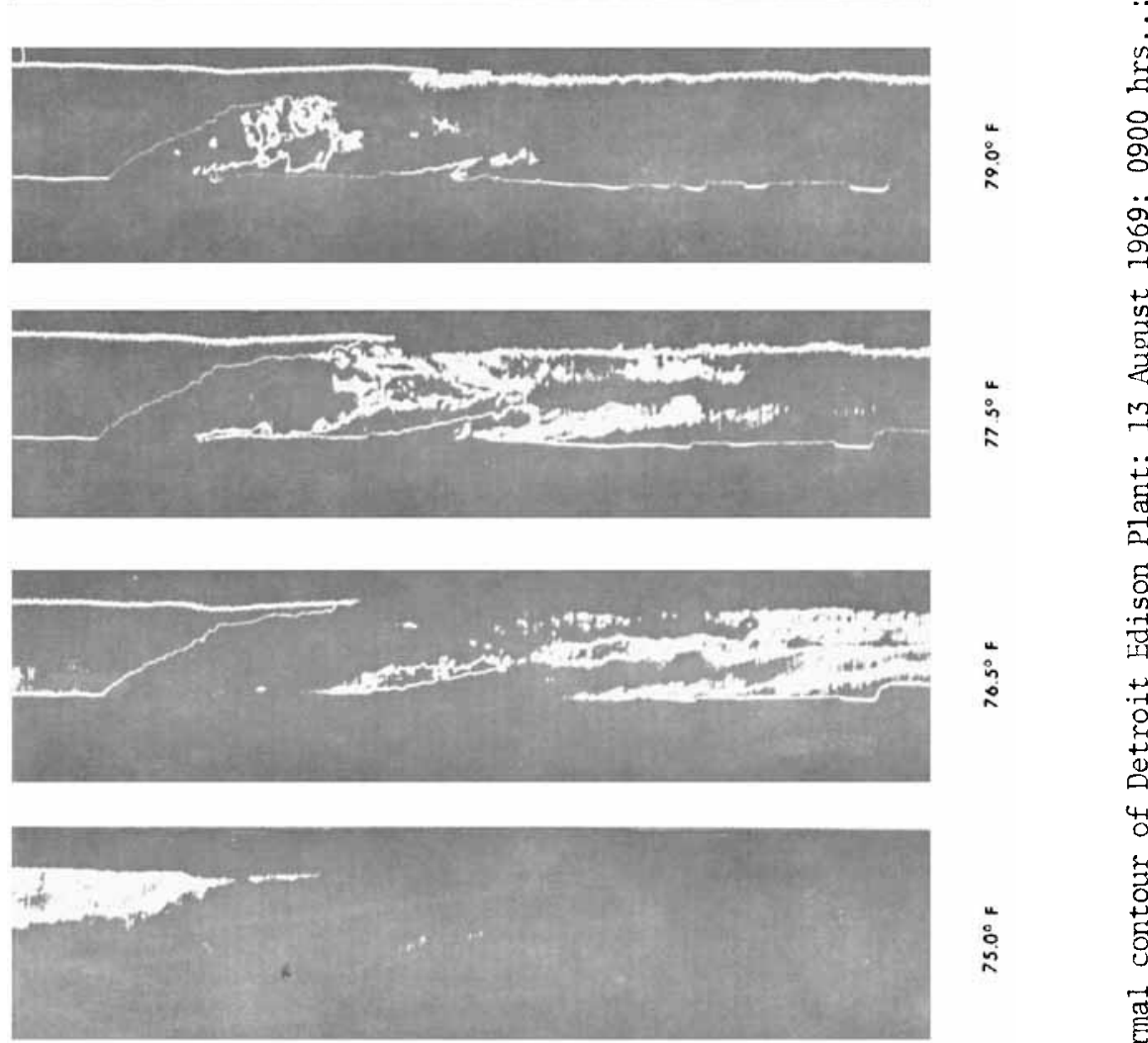

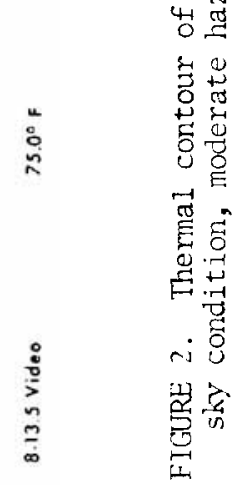



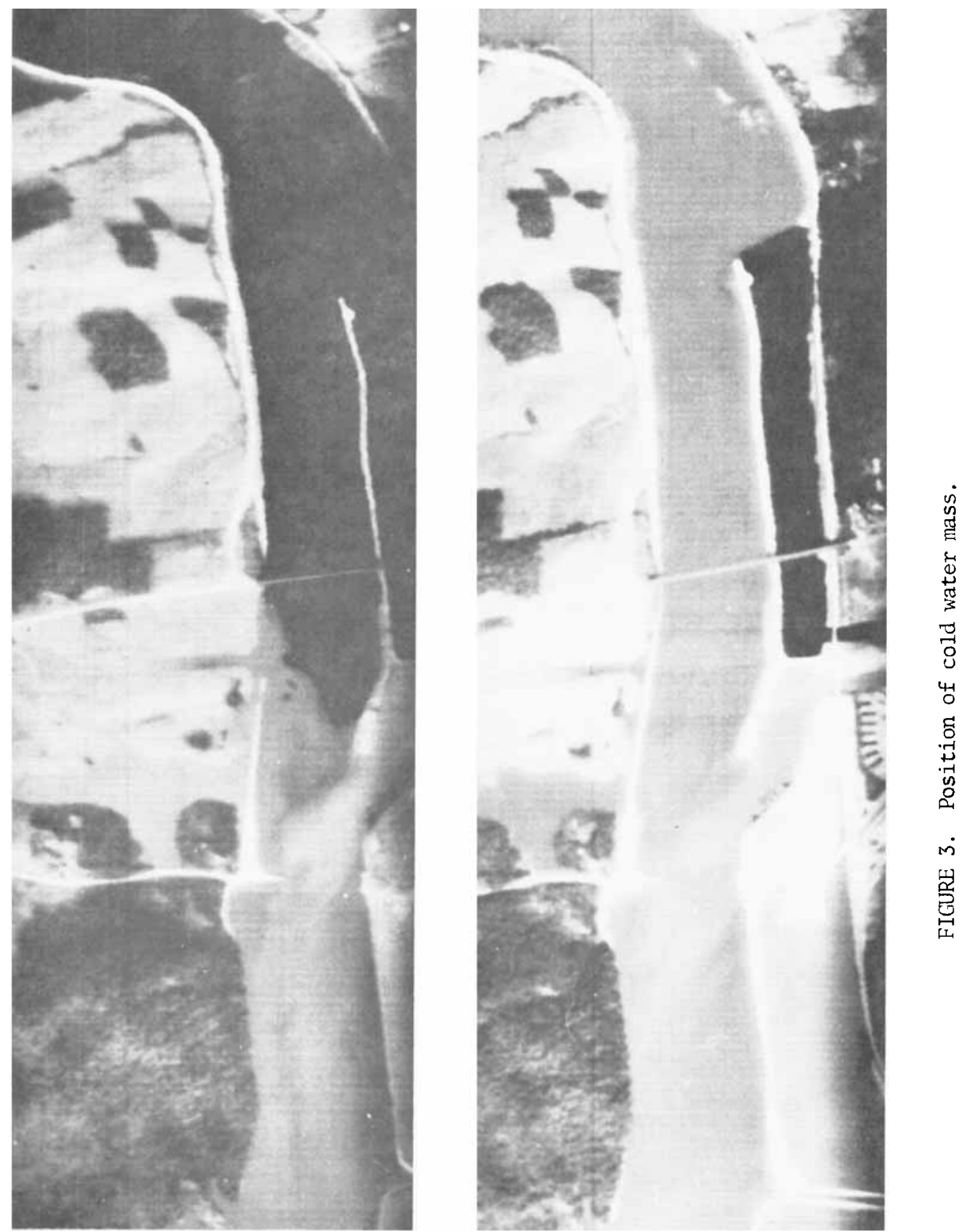


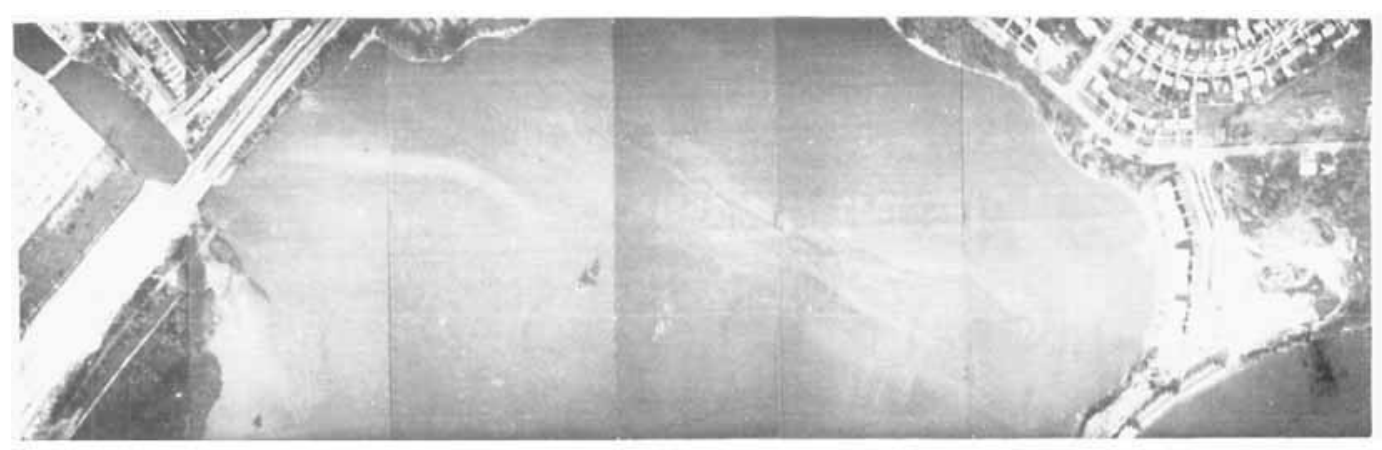

\section{Conventional Photo Mosaic}

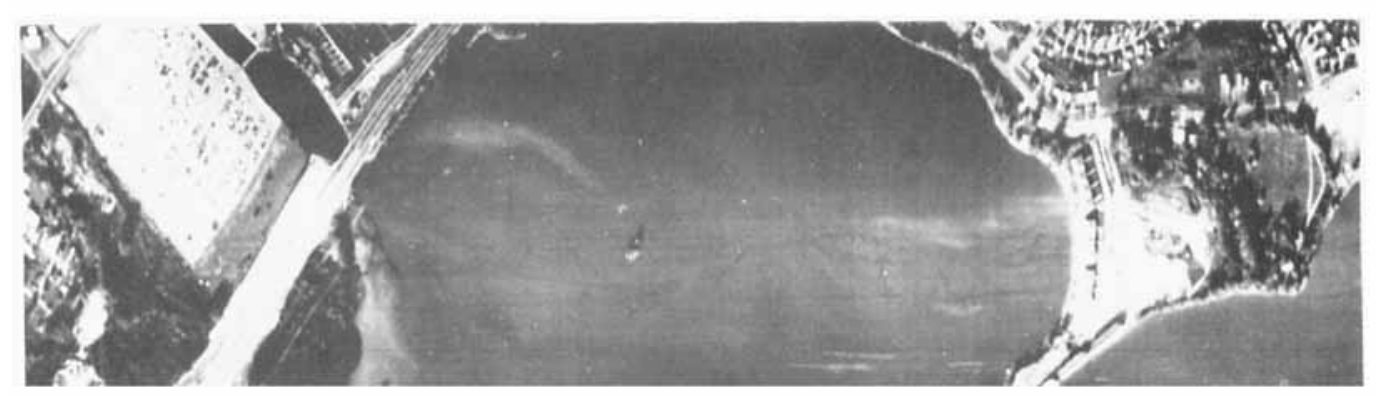

\section{Narrow Band Scanner Imagery 0.55 to $0.58 \mu$}

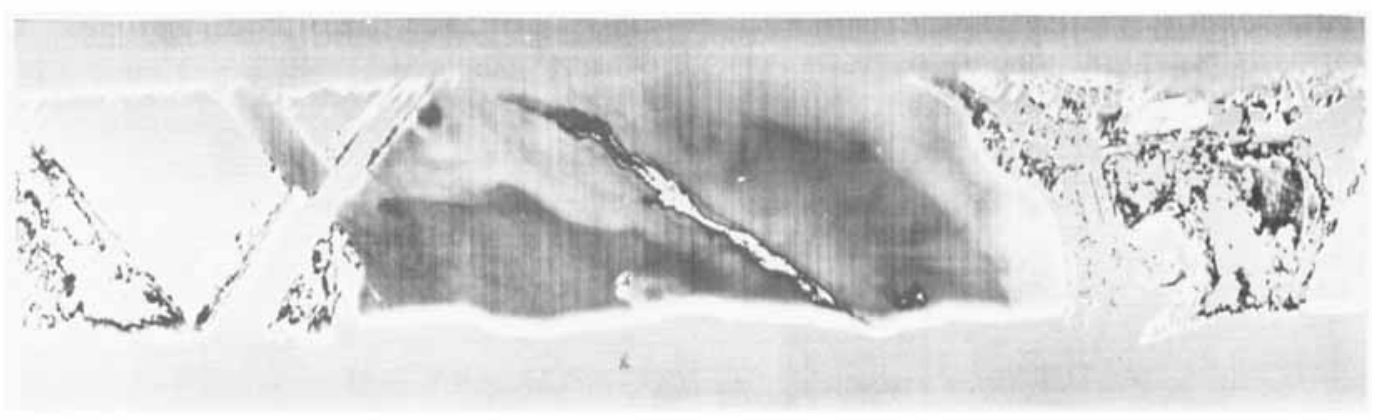

Enhanced Scanner Imagery 8.0 to $14.0 \mu$

FIGURE 4. Municipal waste treatment plant discharge. 

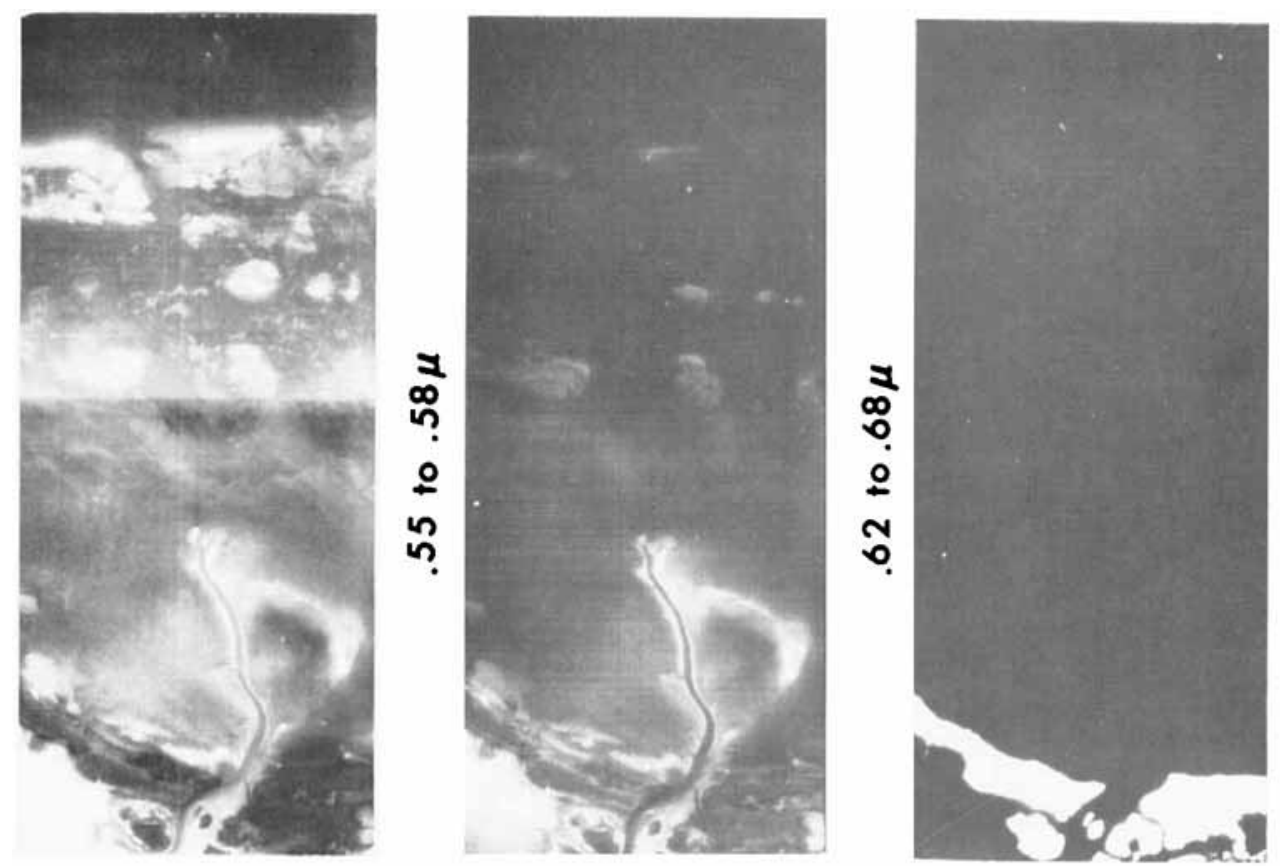

$\frac{1}{8}$
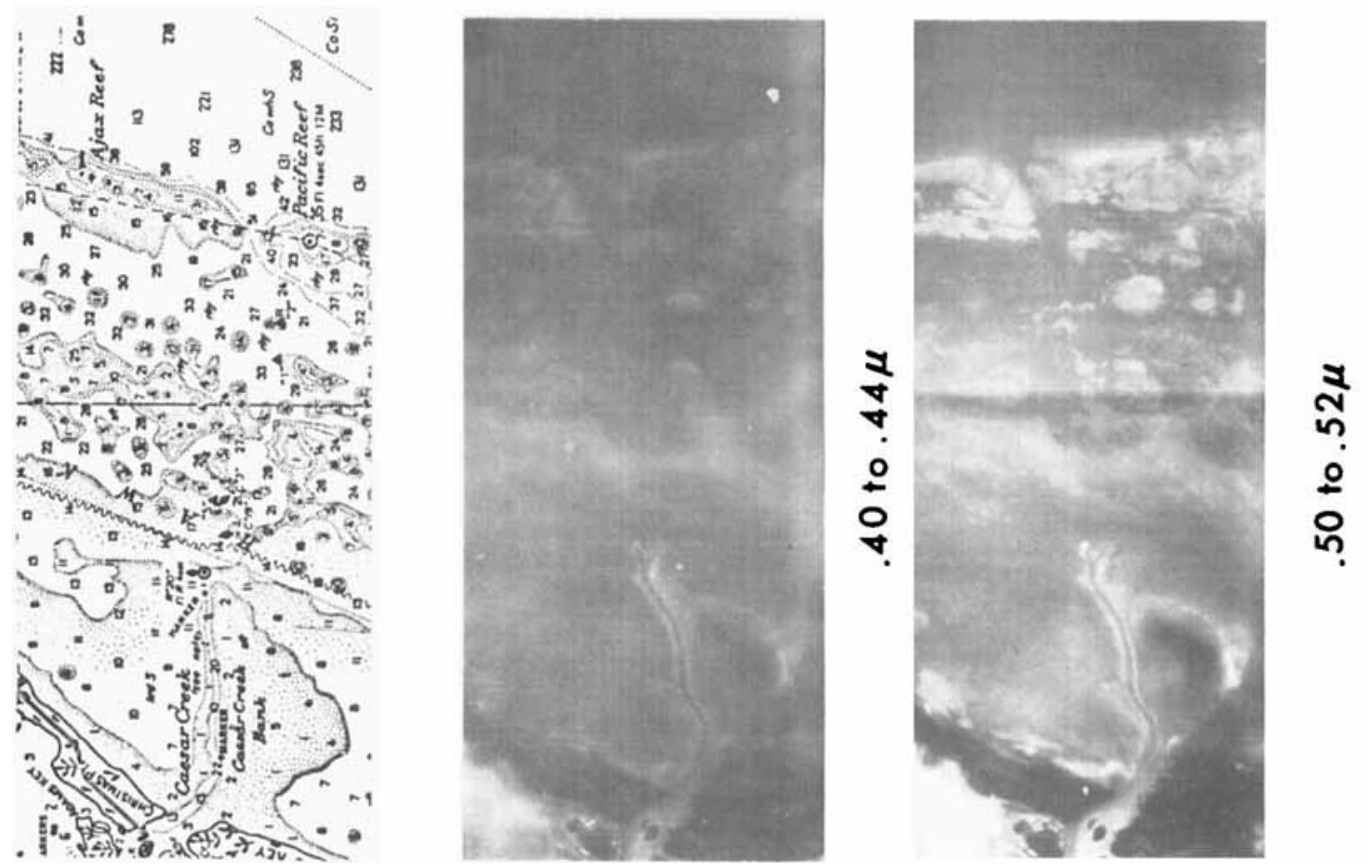

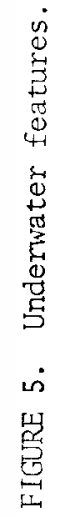



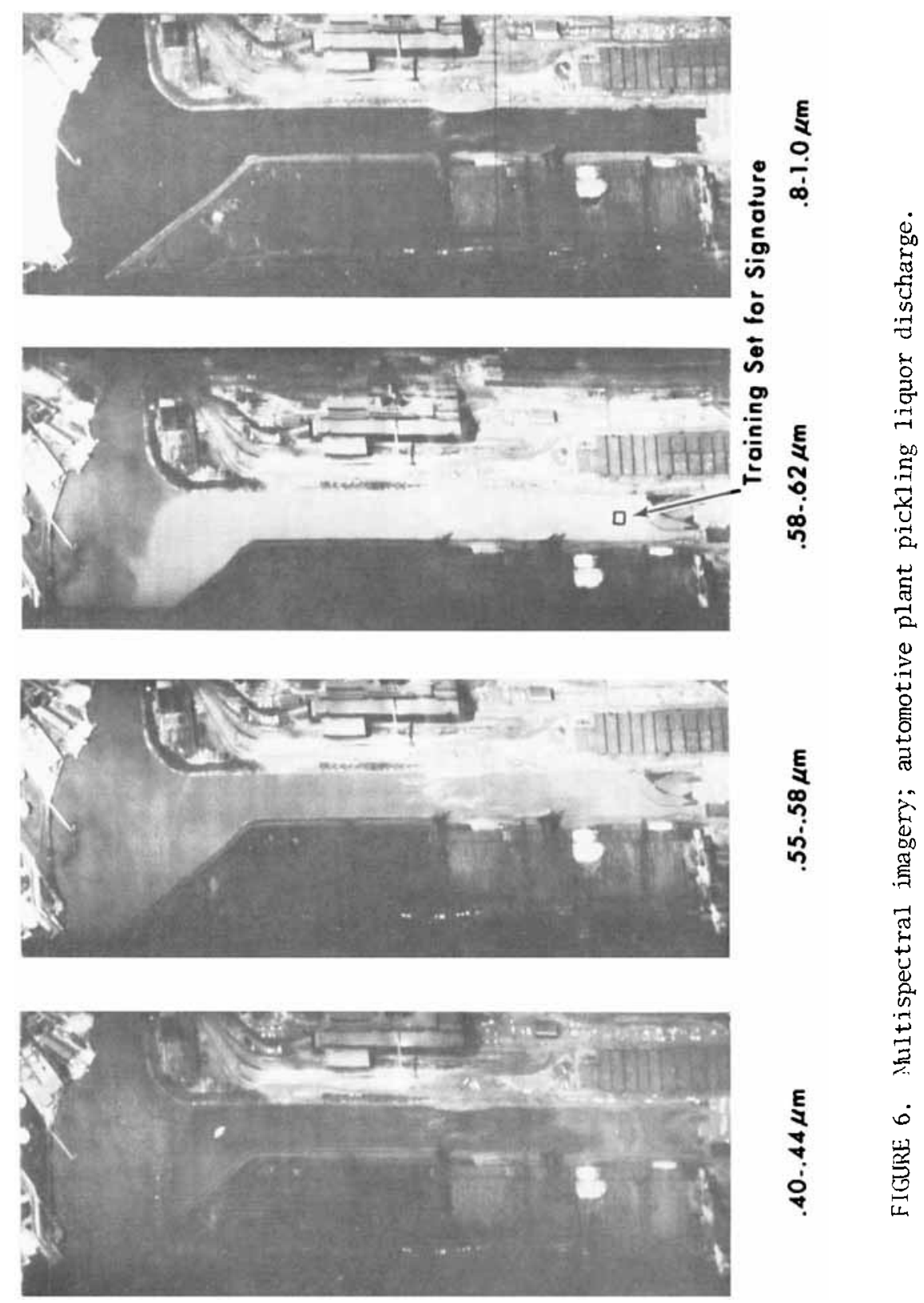

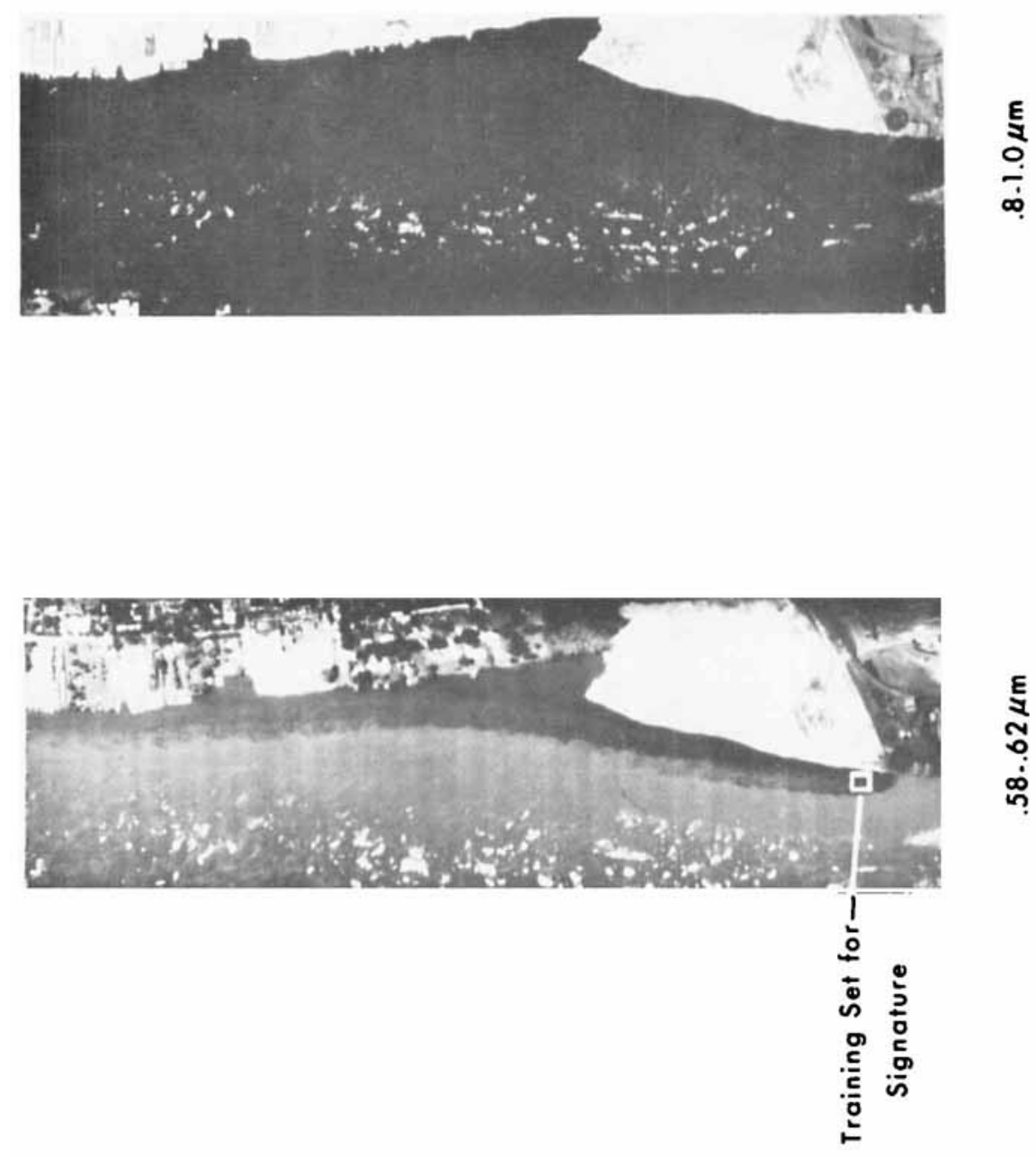

$\frac{E}{0}$

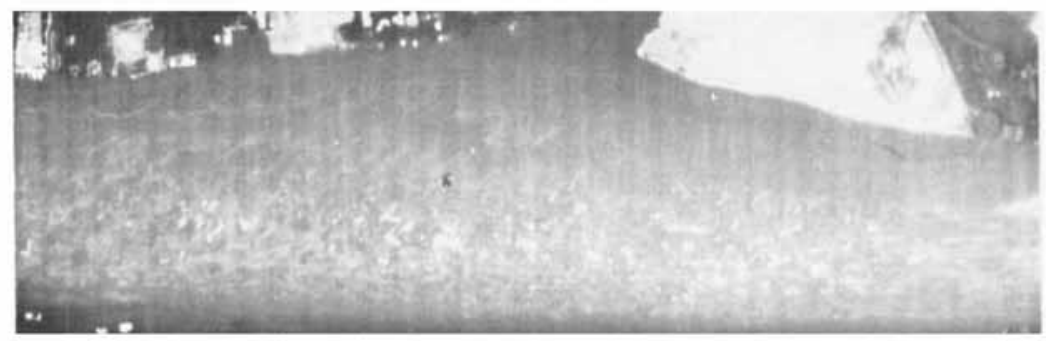

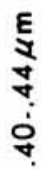




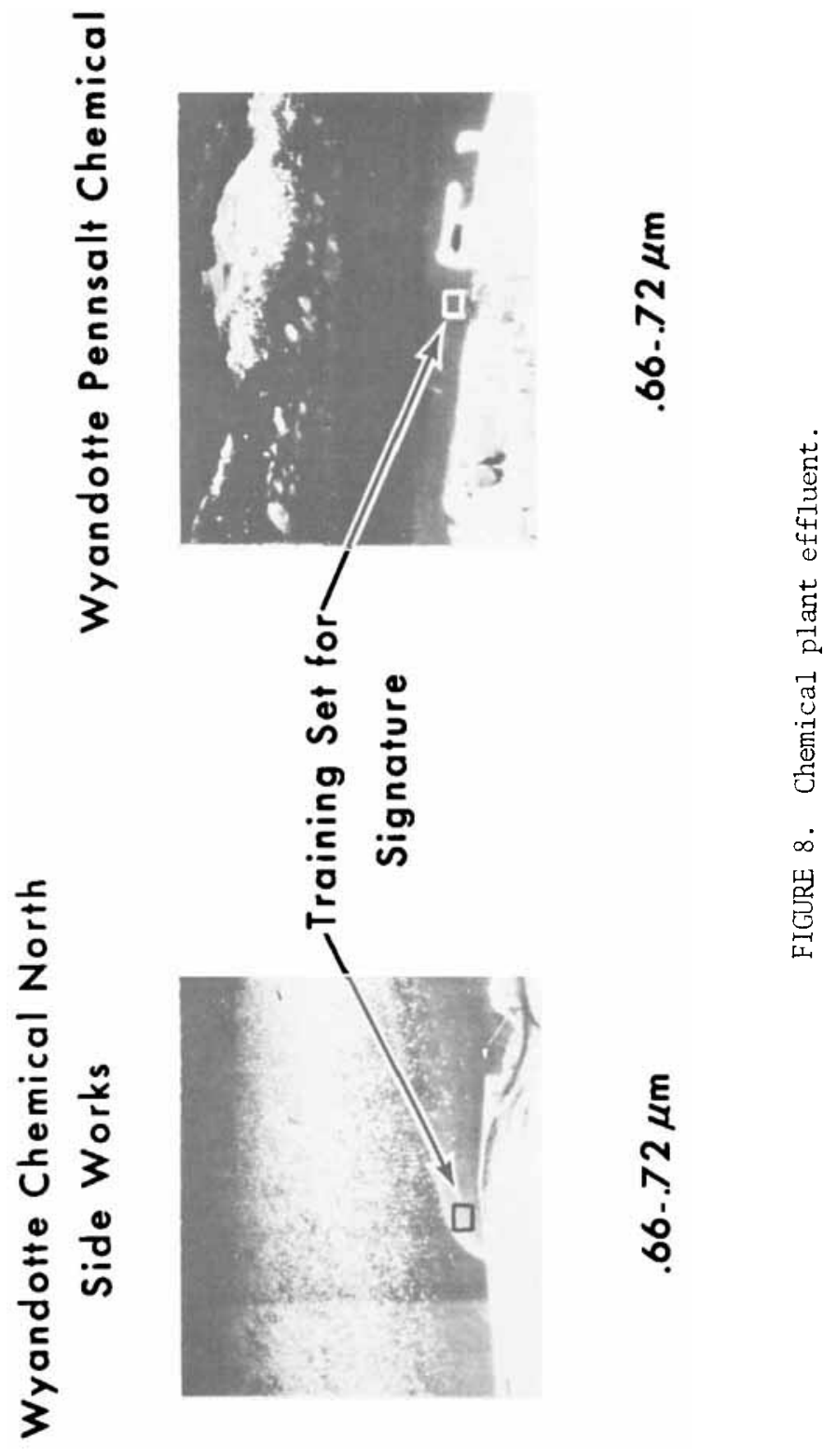




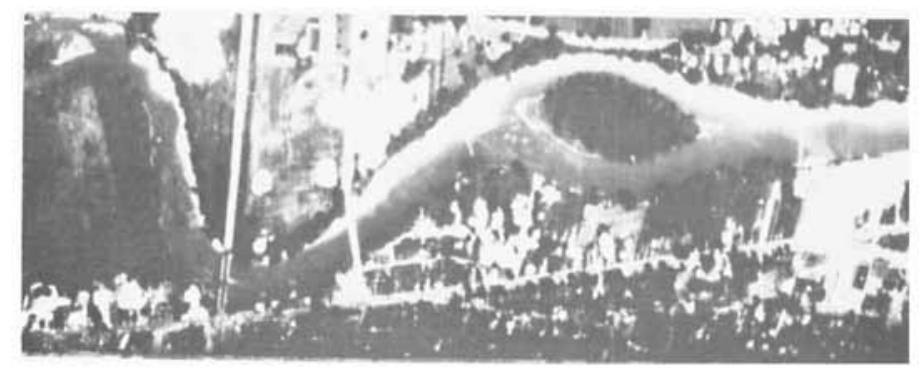

ลัฐ
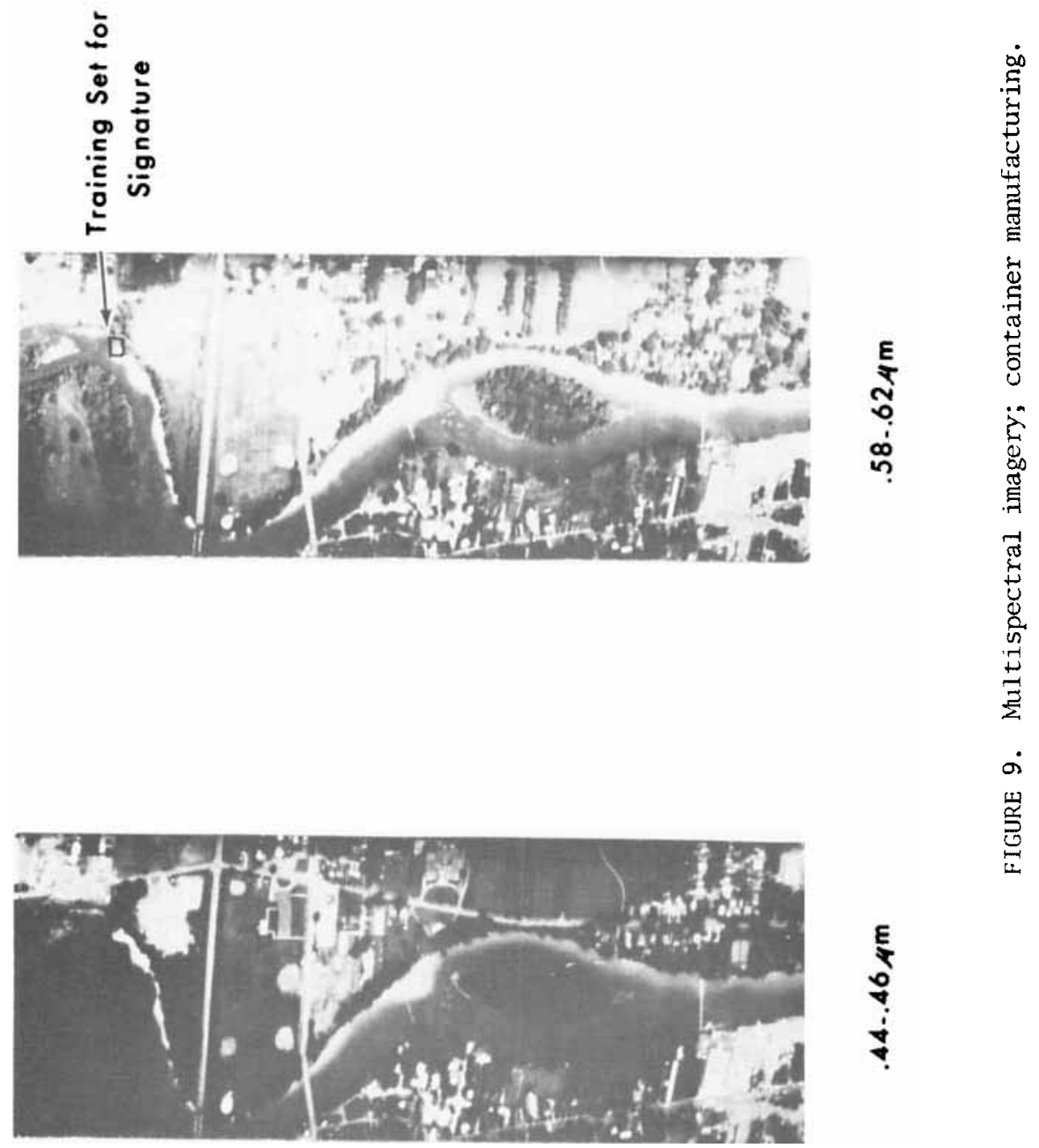

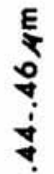


Inspection of the above figures shows that each effluent is more clearly defined by examination at its optimum wavelength; and, hence, dispersion patterns of the effluent are best determined within a particular spectral band width.

Using the multispectral scanner concept, the optimun wavelength is automatically determined; and in addition, the interactions of radiation with the effluent at other positions in the electromagnetic spectrum are also determined. This is shown in Figures 10 and 1I. Each effluent type and receiving body of water has its unique spectral characteristics which serve to differentiate it from other sources.

It is clear that when a photon of energy strikes a liquid, one of the inter actions which occur is scattering. The selective scattering which takes place is, of course, affected by the concentration, shape, and size of suspended solids. $A$ decrease in concentration will result in a decrease in back-scattered light. ligure 12 illustrates this point. The degree of back-scattering is reduced at a position $100 \mathrm{ft}$. below the outfall in the effluent plume; as well as in an adjacent area in the Detroit River. In this particular case, the optimum spectral position for defining variations in suspended solids concentration is about $.64 \mu$. Using the computer techniques referred to earlier, suspended solids contours could be easily developed. Work is currently in progress to further explore the techniques of suspended solids measurement using the methods described together with other instrumental concepts.

\section{OTHER NONCONTACT MEASUREMENT TECHNIQUES}

The present report describes one instmment and one approach to the problem of measurements in the aquatic environment. This instrumental technique wil1 undoubtedly be further refined and expanded in the near future. However, it is clear that all measurement needs cannot be fully realized using this approach, and that additional concepts will have to be explored. The authors are currently engaged in

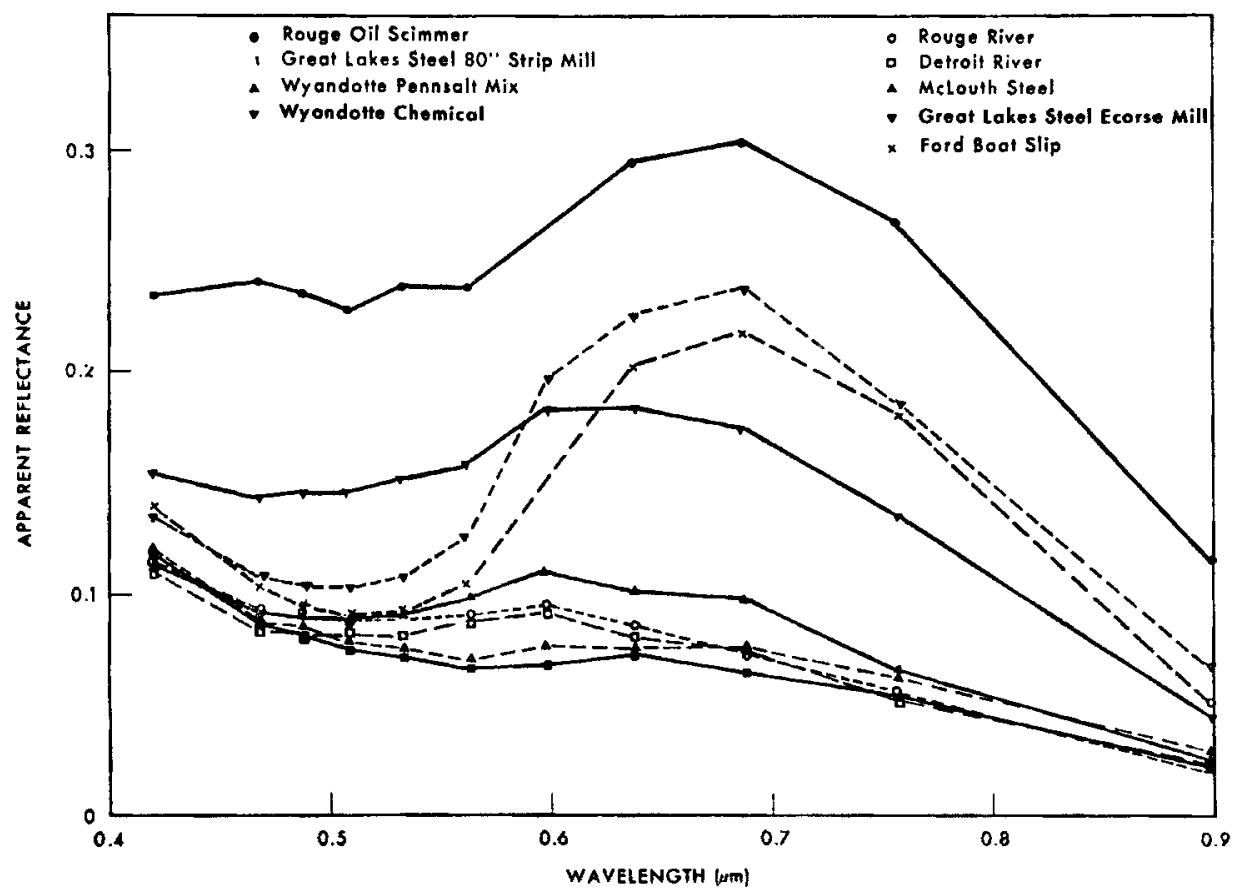

FIGURE 10. Spectra comparison: Rouge and Detroit Rivers. 


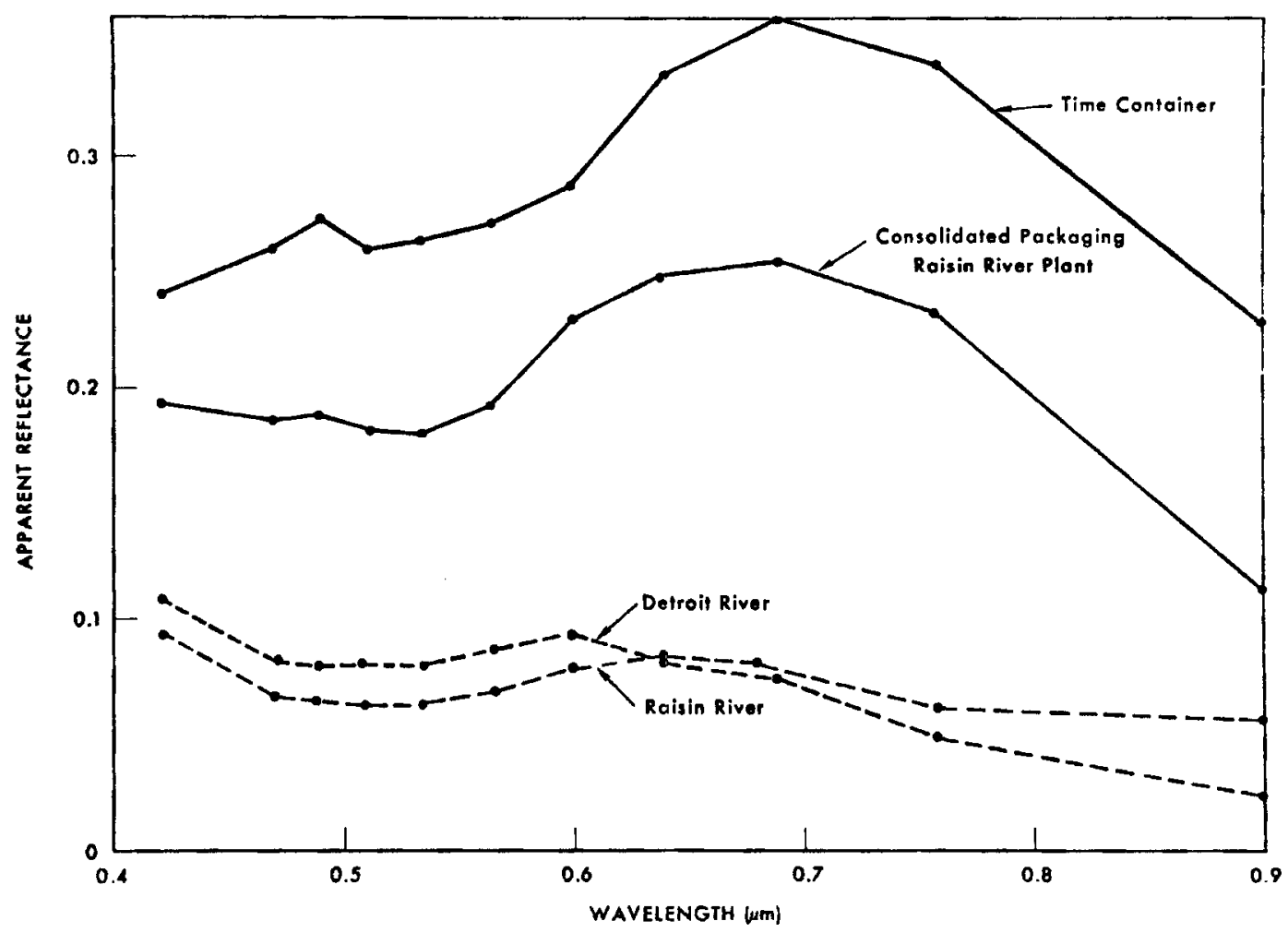

FIGURE 11. Spectra comparison: Raisin River.

laboratory studies dealing with noncontact measurements of specific chemical constituents. One technique which is certain to be used extensively in the future for noncontact data acquisition involves analysis on the basis of fluorescence. This analytical technique could be used for the detection and identification of substances such as rhodamine dye, chlorophyl1, lignin-sulfonates, and many other substances which possess the property of fluorescence. Recent reports which describe the Fraunhofer line discriminator (Stoertz, et al., 1969) and laser induced emissions (Hickmann and Moore, 1970) indicate that airborne fluorescence measurements are feasible.

\section{CONCLUSIONS}

The added sophistication which the multispectral system provides in terms of range of spectral discrimination and electronic processing, clearly indicates that this system can be used to collect a wide variety of data which is of significance in water pollution control and water quality evaluation. As a result, the preliminary data collected to date encourages a continued research effort to fully define the capabilities of this system and related instrumentation. Research is needed to develop spectra for a wide range of pollutants and substances, to define the limits and extent of ground control required, to define the limitations of the system, and to evaluate a number of environmental factors. Additionally, other instrumental concepts must be considered including the use of "active" systems.

The field of remote sensing is still very much in its infancy; however, the prospects are very exciting. The advantages which a fully developed remote sensing technology could offer the field of water quality management are obvious. Speed of 


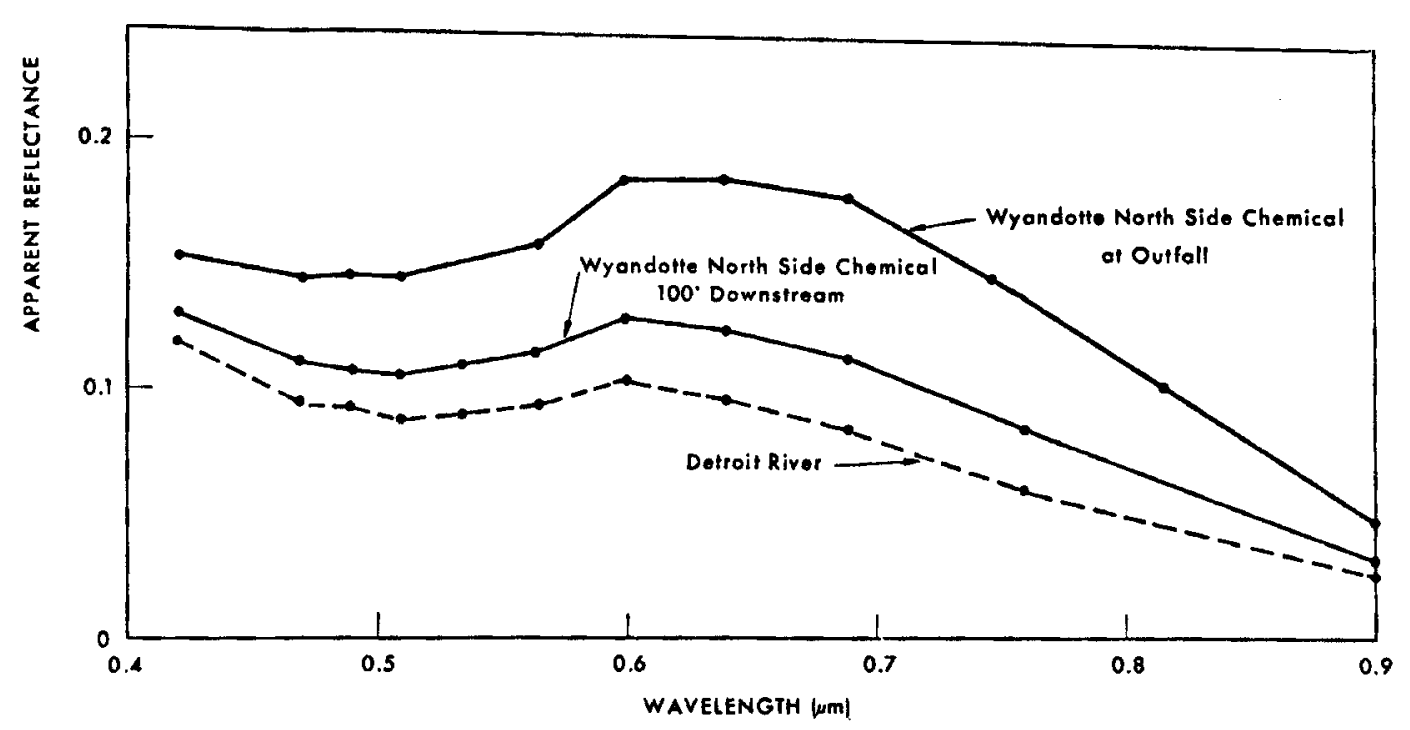

FIGURE 12. Spectral variation due to variation in suspended solids concentration.

data acquisition, mobility, accessibility, and large area coverage, are some characteristics of an airborme system which could provide a new dimension in data acquisition. As a consequence, it is a virtual certainty that in the near future remote sensing techniques will be used for pollution monitoring, study of dynamic conditions in the aquatic environment, and other large scale water resource investigations.

\section{ACKNOWLEDGEMENTS}

This investigation was supported in part by the NSF Sea Grant Program at the University of Michigan. The assistance of Mr. P. Hasell and Mr. S. Stewart in data collection and data reduction is greatly appreciated.

\section{REFERENCES}

Colwel1, R. N., et al. 1963. Basic matter and energy relationships involved in remote reconnaissance. Photogrametric Engineering 29(5):761-799.

Hickmann, G. D. and R. B. Moore. 1970. Laser induced fluorescence in rhodamine B and algae. Presented at the 13th Conference on Great Lakes Research, Buffalo, New York.

Holter, M. R. 1967. Infrared and multispectral sensing. Bioscience $17(6): 376-383$.

Nalepka, R. F. 1970. Investigation of multispectral discrimination techniques. Final Report No. 2264-12-F, Willow Run Laboratories, Institute of Science and Technology, The University of Michigan, Ann Arbor, Michigan.

Polcyn, F. C. and R. A. Rollin. 1969. Remote sensing techniques for the location and measurement of shallow water features. Report No. 8973-10-P, Willow Run Laboratories, Institute of Science and Technology, The University of Michigan, Ann Arbor, Michigan.

Stoertz, G. E., W. R. Hemphi11 and D. A. Markle. 1969. Airborne fluorometer applicable to marine and estuarine studies. Marine Technology Society Journa1 3(6): $11-26$. 